\author{
Was ist neu in der medikamentösen Therapie? Wir halten Sie auf dem Laufenden \\ mit Berichten von Kongressen und Symposien der pharmazeutischen Industrie.
}

\title{
Immuntherapie beim malignen Melanom
}

\section{Ein Drittel der Patienten überlebt fünf Jahre}

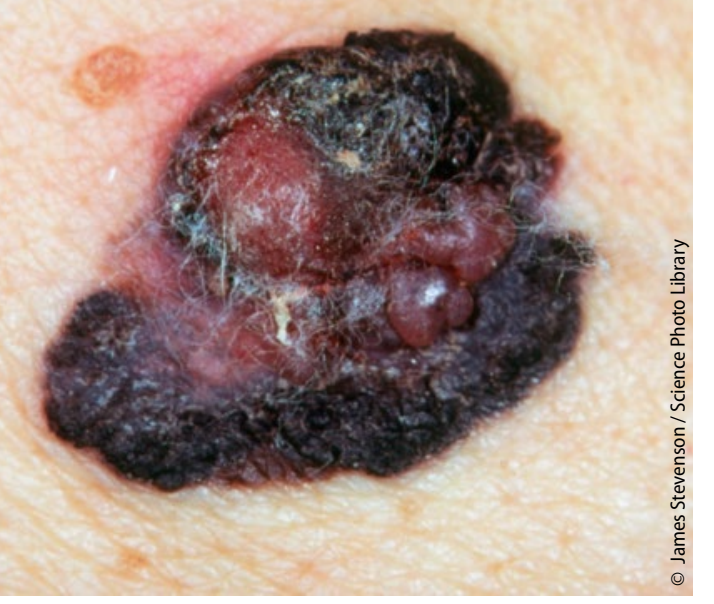

Die Diagnose malignes Melanom ist nicht mehr so vernichtend wie ehedem.
_ Neuen, beim Kongress der American Society of Clinical Oncology (ASCO) 2016 präsentierten Studiendaten zufolge hat ein Drittel der Patienten mit malignem Melanom im fortgeschrittenen, metastasierten Stadium dank Checkpoint-Inhibitoren Aussicht auf ein Langzeitüberleben.

Die KEYNOTE-006-Studie verglich bei 834 Patienten den PD-1-Inhibitor Pembrolizumab (Keytruda ${ }^{\circledR}$ ) mit Ipilimumab. 36\% der Patienten sprachen auf Pembrolizumab, 13\% auf Ipilimumab an. Die mediane Dauer des Ansprechens war nach zwei Jahren noch nicht erreicht: 55\% der Patienten in der Pembrolizumab-Gruppe und 43\% in der Ipilimumab-Gruppe überlebten zwei Jahre.

In der KEYNOTE-029-ExpansionKohorte wurden Patienten mit Pembro- lizumab (Normaldosis) plus Ipilimumab (niedrige Dosis) behandelt. Die Ansprechrate betrug $57 \%$ und lag somit beträchtlich höher als in den Monotherapie-Studien. 10\% zeigten eine komplette Remission.

Prominentester Melanompatient unter Pembrolizumab ist der 91-jährige ehemalige US-Präsident Jimmy Carter. Zeitungsberichten zufolge hatte er bereits Leber- und Hirnmetastasen, seine Prognose wäre vor fünf Jahren infaust gewesen. Heute ist er nach einer Pembrolizumab-Behandlung tumorfrei in Vollremission. Die Behandlung wurde ausgesetzt.

Dr. Dirk Einecke

- Mittagsseminar "Immuncheckpoint-Blockade beim Melanom", 25. Fortbildungswoche für parktische Dermatologie und Venerologie; München, Juli 2016 (Veranstalter: MSD)

\section{Antiinfektivum überzeugt in aktueller Studie}

_ Erst ein Kratzen im Hals, dann Hustenreiz und Schnupfen, oft begleitet von Kopf- und Gliederschmerzen - die typischen Symptome eines grippalen Infekts kennt jeder aus eigener Erfahrung. Die Symptome schränken das Alltagsleben deutlich ein und können zu erheblichen Fehlzeiten in Schule und Beruf führen. Contramutan $^{\circledast} \mathrm{N}$-Saft, ein Antiinfektivum mit Extrakt aus dem echten indianischen Wasserdost, ist in der Lage, die akuten Erkältungssymptome rasch zu lindern.
Die Wirksamkeit und Anwendungssicherheit dieser Wasserdost-Zubereitung wurde in einer großen nicht-interventionellen Studie untersucht, an der u. a. 418 Jugendliche (ab zwölf Jahren) und Erwachsene teilnahmen, deren Erkältungssymptome im Mittel seit 34,7 Stunden bestanden hatten. Die Patienten nahmen das pflanzliche Antiinfektivum im Durchschnitt 7,4 Tage lang ein.

Im Anwendungszeitraum nahmen alle Leitsymptome des akuten grippalen Infekts (Fieber, Halsbeschwerden, Kopf- und Gliederbeschwerden sowie Schnupfen) deutlich ab. Die behandelnden Ärzte beurteilten die Wirkung bei 83,5\% der Patienten als „sehr gut“ und "gut“.

In der Gruppe der Jugendlichen und Erwachsenen wurden nur neun (2,15\%) unerwünschte Arzneimittelwirkungen dokumentiert. Diese waren nur leicht bis moderat ausgeprägt und besserten sich rasch wieder.

\section{Red.}

- Michalsen A, Uehleke B, Stange R. Regul Toxicol Pharmacol. 2015;72:179-84 